should be made to develop and standardize minimum fitness standards for responders. By mitigating the risk of illness or injury to disaster responders, the likelihood of mission success and provider wellness can be increased.

Prehosp Disaster Med 2017;32(Suppl. 1):s57-s58

doi:10.1017/S1049023X17001583

\section{Review of Instruments Used in Hazard Vulnerability} Analysis of Hospitals Douglas A. Romney ${ }^{1}$, Meg S. Femino ${ }^{2}$, Ritu R. Sarin ${ }^{1}$, Michael S. Molloy ${ }^{1}$, Amalia Voskanyan ${ }^{1}$, Gregory R. Ciottone ${ }^{1}$

1. Department Of Emergency Medicine, BIDMC Fellowship in Disaster Medicine, Boston/MA/United States of America

2. Emergency Management, Beth Israel Deaconess Medical Center, Boston/MA/United States of America

Study/Objective: To perform a qualitative comparison of instruments used for hazard vulnerability analysis of hospitals. Background: Analysis of hazard vulnerability is the process by which a hospital determines the relative priority of each potential threat to the organization when allocating resources for disaster preparation and mitigation. While all hospitals in the United States are required to perform a hazard vulnerability analysis annually and use their findings to guide planning efforts, no officially sanctioned instrument exists for this task. Thus, a variety of tools exist in the public domain to assist hospitals in analysis of hazard vulnerability.

Methods: Hazard vulnerability analysis instruments were identified using a standardized online search technique. For each instrument, we compare the hazards identified for analysis, the method of determining probability, magnitude, and mitigation for each hazard, as well as the method used to determine risk using qualitative methodology.

Results: This study is in progress, with results expected by December 2016.

Conclusion: The study is currently ongoing. We anticipate that instruments will vary significantly in the specific threats assessed, calculation of probability, and measure of severity. Relative strength and weaknesses of different instruments will be highlighted. It is of concern that the hazard vulnerability analysis of hospitals in the United States may be skewed by the specific instrument chosen, and that no recommendations currently exist to guide the efforts of emergency managers. Our hope is that this review of available instruments will lead to further research into best practices, resulting in the standardization of the hazard vulnerability analysis of hospitals in the United States.

Prehosp Disaster Med 2017;32(Suppl. 1):s58

doi:10.1017/S1049023X17001595

\section{Modern Strategies of Collaborating Centers for}

\section{Emergencies}

Gennady Kipor ${ }^{1}$, Sergei Goncharov ${ }^{2}$, Natalia Pichugina ${ }^{1}$

1. International, All Russian Center for Disaster Medicine "Zaschta", Moscow/Russian Federation

2. Directorat, All Russian Center for disaster Medicine "Zaschita", Moscow/Russian Federation.
Study/Objective: The main goal is an analysis of their particular activities and tools of coordination.

Background: The number of Collaborating Centers in Disaster Medicine working under the leadership of the World Health Organization (WHO) has been reduced. There are only seven centers that have been accredited by WHO - Great Britain and France (joint Center), Switzerland, Italy, Indonesia, Netherlands, Russia, and the US. Twenty years ago, there were approximately 20 Collaborating Centers in Emergencies.

Methods: Analysis of Disaster Medicine Collaborating Centers and issues addressed at their annual meetings.

Results: Main functions of the promoted Centers: GB and France Center - Support of secretariat for certification; registration and training of international emergency medical teams; providing sustainability and preparedness in vulnerable countries; crisis management. Switzerland Center: All problems of refugees and temporary displaced persons. Italian Center: Support of health system resistance to emergencies, disasters, and crisis. Center develops four programs of research activities and four programs of education - training. Center implements a complex program of evaluation in 15 Italian hospitals. Center proposed a system of distant computer education and training, opened for all registered users. This virtual software lends itself to play out practically any intervention in any scenario of emergency. Netherland Center: On-site courses of education, postgraduate education; analysis of national health strategies in emergencies; information sharing and distribution of information about health systems in developing countries. Indonesia: Program of crisis management in emergencies and in large scale disasters - floods and earthquakes. Every year, all collaborating centers arrange a joint coordinating meeting for information sharing and for arranging bilateral and multilateral agreements for their future activities.

Conclusion: All collaborating centers participate in the WHO International Programs. There is no collaborating center in Africa or in the Extreme Orient. The US Center realizes its activities in isolation from the network.

Prehosp Disaster Med 2017;32(Suppl. 1):s58

doi:10.1017/S1049023X17001601

Understanding the Emergency Preparedness Programs of Academic Health Systems

Katherine Kemen ${ }^{1}$, David Reisman ${ }^{2}$, Robert Seger ${ }^{2}$, Julia Sinclair ${ }^{3}$, Ann Prestipino ${ }^{4}$, Barry Wante ${ }^{5}$, Eric Goralnick ${ }^{5}$, Paul D. Biddinger ${ }^{1}$

1. Emergency Preparedness, Partners HealthCare System, Boston/ MA/United States of America

2. Center For Disaster Medicine, Massachusetts General Hospital, Boston/MA/United States of America

3. Administration, Brigham and Women's Hospital, Boston/MA/ United States of America

4. Administration, Massachusetts General Hospital, Boston/MA/ United States of America

5. Emergency Management, Brigham and Women's Hospital, Boston/MA/United States of America

Study/Objective: We surveyed US academic health systems to understand structure, functions of, and resources dedicated to system-level emergency preparedness (EP) programs. 
Background: In recent years, US hospitals have dedicated significant resources to improve their EP, especially following September 11, 2001. Over the same period, cost containment pressures and consolidation within the US health care system had lead to more hospitals owned by single parent organizations. As hospitals are under continued pressure both to be ready for disasters, and to maximize value, there is limited data describing the role of the system's administrative organization in supporting the preparedness of their hospitals.

Methods: We developed and administered a survey regarding health systems' EP efforts to 97 academic health systems. Data gathered included program funding, governance, preparedness and response roles, and resources provided to system members Results: Of the 38 responding health systems, $87 \%$ were nonprofit. Median revenues were nearly $\$ 2.5 \mathrm{~B}$ USD. Systems had a median of 16,500 employees and nine member entities. 74\% reported having system-level EP staff. $24 \%$ had an annual operating budget of $\$ 100,000-\$ 1 \mathrm{M}$. Most frequently occurring activities included: creating plans, trainings, or exercise templates (68\%); providing access to subject matter experts (68\%); promoting staff preparedness (68\%); and developing plans $(66 \%)$. We identified discrepancies between respondents' descriptions of the resources their system provides for member entities compared with resources they believed should be provided.

Conclusion: Currently, there is wide variation in the resources, capabilities, and programs supporting EP at the system-level among academic health systems. The most common systemlevel resources provided to system entities include a massnotification system, subject matter expertise during planning and emergencies, centralizing emergency supply contracts, and providing support for training and exercises. It is unknown which of these systems and resources may be most needed and/ or most effective, as outcome data has not yet been collected.

Prehosp Disaster Med 2017;32(Suppl. 1):s58-s59

doi:10.1017/S1049023X17001613

\section{Emergency Services Rapid Assessment Tool in}

San Salvador, El Salvador

Eric Cioe-Pena ${ }^{1}$, Nicholas Fling ${ }^{2}$

1. Emergency Medicine, Northwell Staten Island University Hospital, Staten Island/NY/United States of America

2. Denver School Of Medicine, University of Colorado, Aurora/CO/ United States of America

Study/Objective: Our study assessed the emergency care system of the hospitals in the San Salvador metropolitan area in El Salvador. San Salvador is the capital and largest city and the epicenter for trauma and emergency care need.

Background: In El Salvador, over 32\% of all deaths are due to trauma, and cardiovascular emergencies are a rapidly rising incidence of both morbidity and mortality. Doctors working in Emergency Wards (EWs) are on the front line of caring for trauma patients. However, emergency medicine training is not yet developed nor standardized.

Methods: This study utilized the SidHARTe Emergency Services Rapid Assessment Tool (ESRAT), which analyzes resources related to emergency care within a hospital. Survey teams went to the 8 public hospitals to interview key stakeholders in the EW as well as hospital administrators. Structured interviews were conducted about hospital capacity and resources, and observations regarding emergency care supplies were recorded. Epidemiological factors such as access to essential supplies, services and medications were determined using simple statistical methods.

Results: A total of 8 hospitals were surveyed with responses obtained from $97.2 \%(70 / 72)$ of the individuals sought. Emergency care in $100 \%$ of hospitals surveyed is free to the patient. As well, $100 \%$ reported consistent electricity, though $37.5 \%$ reported inconsistent access to running water. All 100\% reported access to all essential lab studies listed in the survey, and reliable access to supplies of blood. Half of EWs surveyed report access to an ultrasound machine, and only $37.5 \%$ report the ability to contact trained staff after-hours. EWs were stocked with, on-average, 60\% (31.9/53) of "Essential Emergency Medicines," 81\% (52/64) of "Essential Emergency Supplies," and $90 \%$ of "Essential Emergency Equipment" (5.4/6).

Conclusion: This survey establishes a baseline capability of the public hospitals in San Salvador, and serves as an important benchmark for the continued development of emergency care resources and services nationwide.

Prehosp Disaster Med 2017;32(Suppl. 1):s59

doi:10.1017/S1049023X17001625

\section{Impact of Participation in Focus Groups on Perceived \\ Preparedness for Emerging Threats \\ Bruria Adini ${ }^{1}$, Avi Israeli ${ }^{2}$, Moran Bodas ${ }^{1}$, Kobi Peleg $^{1}$}

1. Disaster Management \& Injury Prevention, Tel-Aviv University, Tel Aviv/Israel

2. Hadassah Hebrew University, Jerusalem/Israel

Study/Objective: To examine the impact of participation in focus groups on perceived emergency preparedness for an emerging threat of attack on civilian populations.

Background: Health care systems are required to develop preparedness for all hazards that risk public health and safety. Policies for managing emerging (newly evolving) threats should be prepared based on multi-disciplinary perspectives that promote an effective and comprehensive response. Focus groups are instrumental in designing policies, but their impact on perceived emergency preparedness has not as yet been presented.

Methods: Five multi-disciplinary focus groups were created to review risk assessment and recommend policies for managing an emerging threat of missile attacks against civilian populations, including: providing community health care services; hospitals' operational continuity; casualty evacuation; continuous medical care to vulnerable populations; and providing medical services in 'closed military zones.' Fifty-nine national and regional managers of the Israeli health care services rotated between the focus groups, recommending applicable policies for all identified challenges. A survey concerning perceived individual and systemic preparedness for the emerging threat was completed pre-post participation in the focus groups. 